\title{
Les matrones équestres de Narbonnaise
}

\author{
- Anthony Álvarez Melero ${ }^{1}$
}

Résumé:

Afin de combler un vide historiographique dans les recherches relatives aux élites provinciales, je me propose d'étudier la parenté féminine des chevaliers romains, pour cerner leur rôle dans la promotion de leurs proches dans les ordines supérieurs.

Mots-clés

Ordre équestre, femmes, mariage, sacerdoces, richesse

Abstract:

The aim of this paper is to fill an historiographical gap related to the research about provincial elites. The purpose is to study the female relatives of Roman knights in order to identify their role in promoting their relatives in the superior ordines.
C Women have their uses for historians. They offer relief from warfare, legislation, and the history of ideas; and they enrich the central theme of social history, if and when enough evidence is available". C'est en ces termes que s'exprimait Sir R. Syme, en page 168 de son Augustan Aristocracy, paru à Oxford en 1986, au début d'un chapitre sur les princesses de la domus Augusta et les femmes présentes à la cour. Le savant d'origine néo-zélandaise entendait par là souligner l'importance de celles-ci dans l'histoire sociale de Rome. De fait, R. Syme poursuit en affirmant que les témoignages les concernant, bien que fortuits et clairsemés (casual and sporadic), nous éclairent sur les alliances matrimoniales, avec leurs préliminaires, les fiançailles, et leurs conséquences, le divorce ou le veuvage, mais aussi sur la licence et la luxure (licence and luxury), la parenté et la discorde (kinship and discord), sans oublier bien évidemment les richesses acquises parfois de longue date par leurs familles.

Si cet énoncé est valable pour les dames de la haute société, apparentées aux membres des ordres supérieurs, dans quelle mesure peut-on y souscrire pour celles issues de catégories sociales relativement moins prestigieuses? Difficile de répondre, car en réalité même les femmes de l'élite, pour ainsi dire, sont très souvent laissées pour compte, et pas seulement dans l'historiographie contemporaine, sauf exceptions. En effet, Asconius s'en plaignait déjà amèrement, quand il chercha en vain le nom de l'épouse de P. Rutilius Nudus et bellemère de L. Calpurnius Piso Caesonianus, consul en 58 avant notre ère (Ascon., Pis., 26). Le même constat doit être posé pour les membres de l'ordre équestre, qui comptent pourtant parmi les catégories sociales privilégiées, même si la place prise dans la documentation les marginalise également, si on les compare aux sénateurs. Nous verrons donc ce qu'il en est pour les matrones équestres de Narbonnaise que je propose d'examiner dans cet article. Il s'agira d'établir dans quelle mesure elles participent à la promotion de leurs proches dans l'ordo equester ou à l'ordo senatorius - s'ils étaient en revanche déjà titulaires du cheval public -, selon quelles modalités et quels en sont les fondements économiques. 
Avant de poursuivre, il convient de rappeler brièvement que les «matrones équestres», telles que je les ai étudiées dans ma dissertation doctorale en cours de publication (Álvarez Melero s.p.), sont les épouses, les sœurs, les filles et les petites-filles de chevaliers, comme il est décrit dans le sénatus-consulte de Larinum, daté de l'an 19 de notre ère $(A E 1978,145)$. Toutefois, l'appellation «matrona equestris» que j'ai choisie pour les désigner n'apparaît pas sur la table de bronze de Larino, mais sur le prolixe compterendu épigraphique des Jeux séculaires de l'an 204 ap. J.-C., où il sert en fait à qualifier les participantes aux cérémonies du 2 juin en fonction, sinon de leur «rang», du moins de leur «statut» (CIL VI, $32329+$ $A E$ 1932, 70 = Pighi 1965, fr. V/V ${ }^{a}, 1.26-29=E A O R \mathrm{I}$, 43). Il ne s'agit donc nullement d'un titre officiel, car les femmes, tout au long de l'existence de l'ordre équestre, ne furent en aucune façon membres de cet ordo, à la différence des clarissimae (RaepsaetCharlier 1987, 1-12).

A ce groupe, j'ai voulu adjoindre les mères et aïeules des chevaliers, puisque nous savons que l'ingénuité sur deux générations de la famille du futur titulaire du cheval public était requise pour accéder à l'ordre équestre, comme nous le dévoile Pline l'Ancien (Plin., Nat., XXXIII, 32). Il n'est certes pas question de femmes dans ce texte, mais j'ai souhaité établir si elles aussi étaient soumises à cette obligation.

Enfin, je dois rappeler que j'ai considéré comme «équestres» non seulement toutes les dames que j'ai évoquées jusqu'à présent, à la condition d'être apparentées à des chevaliers qui en font clairement état («simples» equites Romani, officiers équestres, hauts fonctionnaires), mais j’y ai aussi compté les préfets des ouvriers, bien qu'aujourd'hui j'ai tendance à les rejeter, car leur appartenance à l'ordre équestre n'est pas assurée (Álvarez Melero 2013; sur la Narbonnaise, voir aussi Sablayrolles 1984 et la contribution de Ségolène Demougin dans ce dossier), à l'instar des adlecti, sauf indication contraire. Dans ce dernier cas, est exclue de ma liste Iulia D. f. Concessa ( $P I R^{2}$ I 659 et FOS 434), épouse du magistrat et officier viennois C. Fulvius Lupus Servilianus (PIR ${ }^{2}$ F 548 et Burnand 2005-2010, n $121 \mathrm{~S} 22$ ), adlecté parmi les sénateurs de rang prétorien par Vespasien. Par ailleurs, en l'absence de toute titulature strictement réservée aux parentes de chevaliers, il s'avère particulièrement difficile de les recenser sans un patient dépouillement des sources, principalement épigraphiques. Il est vrai que certaines femmes apparentées aux chevaliers sont parfois qualifiées de honestae/honestissimae voire stolatae mais, à moins d'éventuelles variantes régionales, un rapide examen de la question mène à la conclusion qu'elles ne furent pas toutes des matrones équestres. Quant à la chronologie, j'ai opté pour la période comprise entre 18 av. J.-C. et le règne de Gallien, parce que ces dates correspondent, respectivement, au moment où Auguste établit la définition juridique de l'ordre équestre et à la fin à la fois des procuratèles et des milices équestres.

Il va sans dire enfin que dans l'historiographie récente, ce thème n'a pas reçu l'attention qu'il mérite. Je pense, par exemple, au dernier grand travail d'envergure sur les membres de l'uterque ordo des Gaules et des Germanies, rédigé par Yves Burnand, qui exclut totalement les femmes, l'empêchant, par la même occasion d'approfondir certaines questions relatives aux alliances matrimoniales ou à l'assise locale des chevaliers et sénateurs (Burnand 2005-2010. Sur ce thème, plus spécifiquement, voir Christol 2009, mais aussi Pflaum 1978; Demougin 1988, 503-552 - traité dans un cadre plus général, tout comme dans Schäfer 2000).

Ainsi donc, d'après mes dépouillements, le nombre de matrones équestres originaires de Narbonnaise est de trente-deux, et elles sont majoritairement datées du $\mathrm{I}^{\mathrm{er}} \mathrm{s}$. ap. J.-C., puisque vingt-cinq ont vécu à cette époque, une est à classer entre le $\mathrm{I}^{\mathrm{er}}$ et le $\mathrm{II}^{\mathrm{e}} \mathrm{s}$., quatre au $\mathrm{II}^{\mathrm{e}} \mathrm{s}$. et deux au $\mathrm{III}^{\mathrm{e}} \mathrm{s}$.

Quoi qu'il en soit, comparées avec les chiffres fournis par Y. Burnand, les matrones équestres font pâle figure, car cent trente-huit chevaliers sont connus dans la province sur toute la période (ou cent trente et un, si j'enlève de sa liste ceux ayant vécu au-delà des limites temporelles que je me suis imposées). Toutes les cités importantes apparaissent, même si la capitale provinciale, Narbonne, n'est étrangement pas celle qui fournit le plus d'attestations. En effet, Vienne et Béziers sont à cet égard les plus riches en données, dans la mesure où elles présentent respectivement sept et six exemples, soit à elles deux un peu moins de la moitié du total, pratiquement tous au ${ }^{\text {er }}$ s., à l'exception de deux matrones viennoises du II ${ }^{\mathrm{e}}$ s. (tabl. 1).

Nous remarquons, à la vue de ce tableau, que pratiquement toutes les cités qui ont fourni au moins un chevalier possèdent aussi une attestation de matrone équestre. Il s'agit, comme il fallait s'y attendre, de communautés privilégiées, détentrices du rang colonial latin ou romain, suite à un octroi par Pompée, César, les Triumvirs, Auguste ou les empereurs du premier siècle de notre ère (Christol $1993=$ Christol 2010, 231-242 et Christol $1999=$ Christol 2010, 105128; Raepsaet-Charlier 1998, 144-148). 


\begin{tabular}{|c|c|c|c|c|c|c|c|c|}
\hline & & Ier s. av.-ap. & I $^{\text {er }} \mathbf{s}$. & I $^{\text {er }}-I^{\mathrm{e}} \mathbf{S}$. & $\mathbf{I I}^{\mathrm{e}} \mathbf{s}$. & II $^{\mathrm{e}}-\mathrm{III}^{\mathrm{e}} \mathbf{s}$. & III $^{\mathrm{e}} \mathbf{s}$. & Total \\
\hline \multicolumn{9}{|c|}{ Gallia Narbonensis } \\
\hline Alba Heluiorum & CL? & & {$[1]$} & & & & & $0[1]$ \\
\hline Antipolis & CL Triumvirs? & & [1] & & [2] & & $1[1]$ & $1[4]$ \\
\hline Aquae Sextiae & CL; CR Auguste & & $2[3]$ & & [3] & & {$[1]$} & $2[7]$ \\
\hline Arausio & CR Triumvirs & & & & $1[1]$ & & & $1[1]$ \\
\hline Arelate & CR César & & 4 [9] & & {$[3]$} & & {$[2]$} & $4[14]$ \\
\hline Baeterrae & CR Triumvirs & & $6[4]$ & & [2] & & & $6[6]$ \\
\hline Carcaso & CL Triumvirs? & & & & [1] & & & $0[1]$ \\
\hline Forum Iulii & CL; CR César & & $1[5]$ & & & & & $1[5]$ \\
\hline Massilia & CFoed. & & & & {$[1]$} & & {$[3]$} & $0[3]$ \\
\hline Narbo Martius & $\mathrm{CR}$ & {$[1]$} & $2[6]$ & & [2] & & {$[2]$} & $2[11]$ \\
\hline Nemausus & CL César & & $2[8]$ & 1 & [9] & & {$[1]$} & $3[18]$ \\
\hline Reii & CL Auguste & & {$[2]$} & & 1 & & & $1[2]$ \\
\hline Ruscino & CL Pompée & & $1[2]$ & & & & & $1[2]$ \\
\hline Tolosa & CR Domitien & & $1[3]$ & & & & & $1[3]$ \\
\hline Vasio & $\mathrm{CL}$ & [1] & $1[8]$ & & [2] & & $1[1]$ & $2[11]$ \\
\hline Vienna & CL; CR César & [1] & $5[29]$ & & $2[10]$ & & {$[1]$} & $7[41]$ \\
\hline Ciuitas ignota & & & {$[1]$} & & & & & $0[1]$ \\
\hline Total par siècles & & 0 [3] & 25 [82] & 1 & $4[36]$ & & $2[12]$ & 32 [131] \\
\hline
\end{tabular}

Liste des cités d'origine des matrones équestres de Gaule Narbonnaise. Abréviations: CFoed.: cité fédérée; CL: colonie latine; CR: colonie romaine. NB: certaines données chiffrées divergent de celles fournies par Y. Burnand, puisque mon cadre chronologique est plus restreint que le sien. En effet, le savant nancéien comptait deux chevaliers de plus au I Ir s. av. J.-C. et datait L. Aponius de Baeterrae à cette époque-là, également. En outre, il attribue au dernier quart du III $s$. trois chevaliers d'Arelate, de Narbo et de Vasio. Hormis ce dernier, présent dans mes listes, les autres ont été exclus de mes tableaux. Je signale aussi qu'entre crochets droits figure le nombre de chevaliers pour la période donnée d'après les recensements d'Y. Burnand.

Il convient maintenant de passer en revue les trentedeux témoignages recueillis (tabl. 2): à la différence des autres provinces occidentales, même les quelques exemples de matrones pratiquement anonymes, nous permettent de tirer des renseignements fort instructifs, démontrant l'intérêt de l'enquête: nous pouvons ainsi affirmer que furent apparentées à des officiers Adgennia Licinilla et sa mère Licinia L. f. Flavilla de Nîmes; Aemilia D. f. Sextina, de Vienne, [Attia M. f. - - -] et sa mère anonyme, d'Arles; Cornelia Tertulla, épouse d'un officier à Béziers ; Domitia Sex. f., mère d'officiers à Aix-en-Provence; Iulia [- - -]a à Arles; Magia Severina, sœur d'un officier de Vaison; Marcia T. f. Taurina, fille d'un officier et ancien préfet des ouvriers; Olia L. 1. Nice, unie à un ancien préfet des ouvriers, devenu officier à Narbonne; Passeria Q. f. [- - - ] petite-fille d'un officier à Vienne; [- - ] Gratina, épouse d'un officier de Vienne, tout comme Ianuaria, tandis que Prima le fut à Béziers et Secundina à Orange.

Pour sa part, Audasia, s'est unie à un procurateur qui fut préalablement officier; Iulia Procilla fut la fille d'un procurateur de Fréjus, tandis que Pompeia Paulina était née d'un haut fonctionnaire d'Arles et Pompeia T. f. Sextina, d'un sous-procurateur et officier de Vienne, sans oublier un couple anonyme formé par un procurateur et son épouse à Béziers.
Ensuite, à un niveau plus humble, Annia [- - - ] et Antonia Titulla, furent mariées à un préfet des ouvriers, tout comme Faltonia Maximilla, connue ainsi que sa fille, Gresia Priscilla, et sa belle-mère, Varia P. f. [- - - ], toutes trois de Béziers; citons aussi Messia M. f. Quarta, d'Aix-en-Provence et [- - -]lia Pompullina, à Vaison.

Mère de «simples» chevaliers (equo publico), furent Coelia Sex. f. Paterna de Riez et Numisia Lucilia d'Antibes, tandis que Porcia T. f. Tutela fut la sœur d'un equo publico et préfet des ouvriers à Vienne.

Enfin, c'est un sacerdoce réservé aux chevaliers, celui de Caenina (Scheid et Granino Cecere 1999, 100 et 151, n 1; FS 3278. Plus récemment $P I R^{2}$ T 316), qui nous permet d'identifier le mari de la matrone anonyme de Toulouse comme tel.

En plus d'avoir possédé le rang équestre, les parents de ces matrones ont aussi géré des fonctions locales, magistratures et sacerdoces, parfois au niveau provincial: ainsi Sex. Adgennius Macrinus, pontife et IIIIvir chargé de dire le droit à Nîmes; C. Valerius Paetus, ayant détenu toutes les magistratures, flamine et pontife à Château-Roussillon; C. Cascellius Pompeianus, IIIvir chargé de dire le droit et préfet des vigiles et des armes à Nîmes; M. Attius Paternus, 


\begin{tabular}{|c|c|c|c|c|c|c|c|c|c|c|c|c|c|c|c|c|c|c|c|c|c|c|c|c|c|c|c|}
\hline 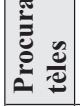 & & & & & & $\underset{x}{\grave{d}}$ & & & & & & $x \mid$ & & & & & & & $x$ & $\frac{m}{x}$ & & & & & $x$ & & \\
\hline 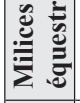 & $\underset{\check{x}}{\bar{m}}$ & $x$ & & & $x$ & $\underset{x}{\Xi}$ & & $\underset{x}{\Xi}$ & $\underset{x}{\Xi}$ & & & & $\left|\frac{\bar{\nabla}}{x}\right|_{x}$ & $\frac{\bar{d}}{x}$ & & & $\frac{\bar{d}}{x}$ & $\underset{x}{\Xi}$ & & $\underset{\check{x}}{\check{x}}$ & & $\left|\frac{a}{x}\right|$ & $x$ & $\mid \vec{x})_{x}$ & & & \\
\hline 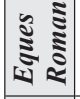 & & & & & & & $\Xi$ & & & & & & & & & $x$ & & & & & $\underset{x}{\Xi}$ & & & & & $\underset{x}{\bar{d}}$ & \\
\hline 施 & $\underset{x}{\Xi}$ & & $\frac{\bar{a}}{x}$ & $\underset{\searrow}{\bar{d}}$ & & & & & 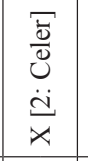 & $\frac{\bar{\beth}}{\rtimes}$ & & & & $\underset{x}{\Xi}$ & $\frac{\bar{d}}{x}$ & & $\frac{\bar{n}}{x}$ & $\underset{\bar{x}}{\bar{x}}$ & & & 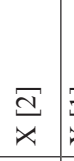 & $\Xi$ & & & & & \\
\hline 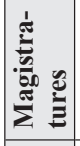 & $\underset{\bar{a}}{\bar{x}}$ & & $\vec{\Xi}$ & $\underset{x}{\Xi}$ & & & $\overline{\frac{a}{x}}$ & $\underset{x}{\grave{\Delta}}$ & 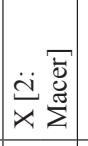 & $\Xi$ & & & 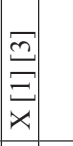 & & $\begin{array}{l}\underset{\mathfrak{\Xi}}{\Xi} \\
\underset{x}{\Xi}\end{array}$ & & $\underset{x}{\Xi}$ & $\underset{\check{d}}{\bar{d}}$ & & $\underset{x}{\Xi}$ & $\frac{\bar{m}}{x}$ & 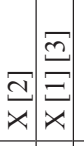 & & \begin{tabular}{|l}
$\bar{\Xi}$ \\
$\bar{x}$
\end{tabular} & & \begin{tabular}{|l|}
$\vec{e}$ \\
$\Xi$ \\
$夭$ \\
\end{tabular} & 3 \\
\hline 总 & $\begin{array}{l}\dot{a} \\
\vec{\Xi} \\
\dot{\Xi} \\
\dot{\sim} \\
\dot{N}\end{array}$ & 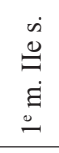 & $\mid \begin{array}{c}\dot{0} \\
\vdots \\
\dot{0} \\
\dot{a} \\
\stackrel{-}{-}\end{array}$ & 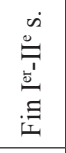 & $\begin{array}{l}\dot{\dot{a}} \\
\stackrel{\Xi}{\Delta} \\
\dot{\Xi} \\
\stackrel{\sim}{\sim}\end{array}$ & $\begin{array}{l}\dot{\dot{D}} \\
\stackrel{\Xi}{ \pm} \\
\dot{\Xi} \\
\stackrel{-}{-}\end{array}$ & $\stackrel{\dot{m}}{\stackrel{\leftrightarrow}{=}}$ & $\begin{array}{l}\dot{\dot{a}} \\
\stackrel{\Xi}{\Xi} \\
\dot{\Xi} \\
\dot{-}\end{array}$ & $\begin{array}{l}\dot{\infty} \\
\dot{\Delta} \\
\dot{a} \\
\dot{a}\end{array}$ & 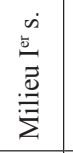 & & & 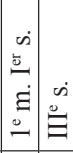 & $\begin{array}{l}\dot{\dot{\Delta}} \\
\stackrel{\Delta}{\dot{g}} \\
\stackrel{\Delta}{\sim}\end{array}$ & $\begin{array}{l}\dot{\Delta} \\
\stackrel{\Delta}{\Delta} \\
\dot{\Xi} \\
\dot{\square}\end{array}$ & $\stackrel{\dot{n}}{=}$ & $\begin{array}{l}\dot{\dot{m}} \\
\stackrel{\Delta}{\dot{g}} \\
\dot{a} \\
\stackrel{-}{-}\end{array}$ & 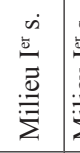 & 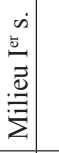 & 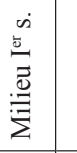 & $\stackrel{\dot{\varphi}}{\varrho}$ & 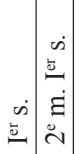 & $\begin{array}{c}\dot{\infty} \\
\dot{\Xi} \\
\dot{\Xi} \\
\stackrel{\Delta}{\sim}\end{array}$ & 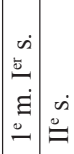 & $\mid \begin{array}{c}\dot{b} \\
\dot{b} \\
\dot{b} \\
\dot{a} \\
- \\
-\end{array}$ & 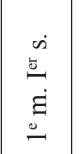 & \\
\hline ש̆ & 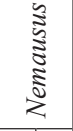 & 气 & 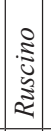 & 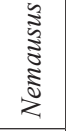 & 華 & 童 & $: \begin{array}{l}: \\
\approx \\
\tilde{z}\end{array}$ & 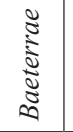 & 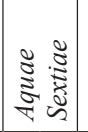 & 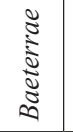 & & | & 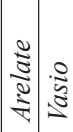 & 吾 & 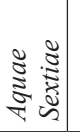 & 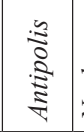 & 童 & $\stackrel{\Xi}{\Xi}$ & | & 离 & 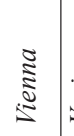 & 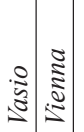 & こ्ञ & 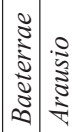 & 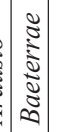 & $\frac{\square}{0}$ & 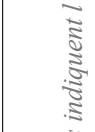 \\
\hline 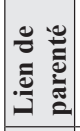 & 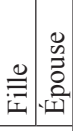 & 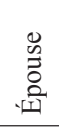 & 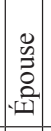 & 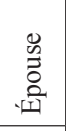 & 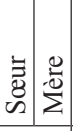 & 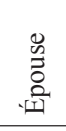 & | & 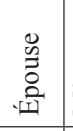 & $\sum_{\substack{\overline{0} \\
\Sigma}}^{0}$ & $\sum^{0}$ & 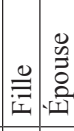 & . & 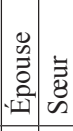 & 釒 & 芦 & \begin{tabular}{|l}
0 \\
0 \\
0 \\
0 \\
.01 \\
.19
\end{tabular} & 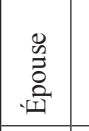 & 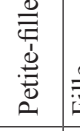 & $\stackrel{0}{\vec{\Sigma}}$ & 兰 & 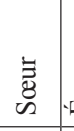 & 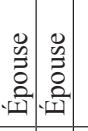 & 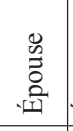 & & & \begin{tabular}{|c|}
0 \\
0 \\
0 \\
0 \\
童 \\
\end{tabular} & 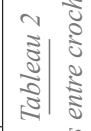 \\
\hline 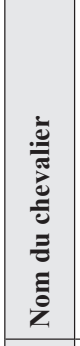 & 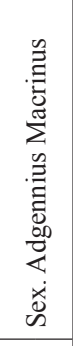 & 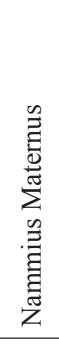 & 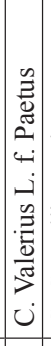 & 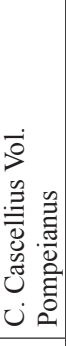 & 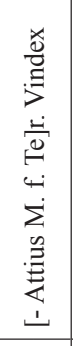 & $\begin{array}{r}\frac{n}{7} \\
\vdots \\
\vdots \\
\end{array}$ & 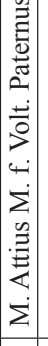 & 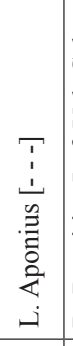 & 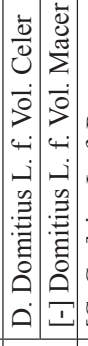 & 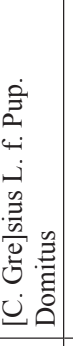 & & 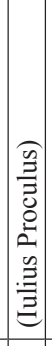 & 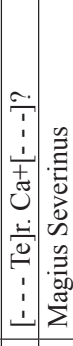 & 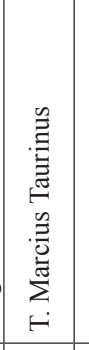 & $\begin{array}{c}\stackrel{0}{Z} \\
\bar{J} \\
心 \\
\vdots \\
\vdots \\
\end{array}$ & 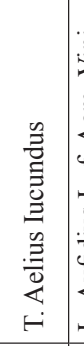 & 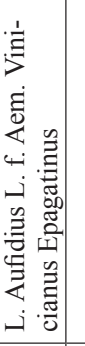 & 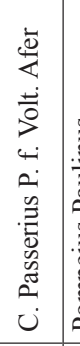 & 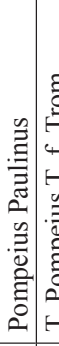 & 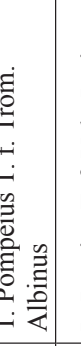 & 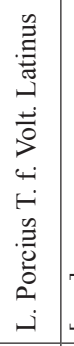 & 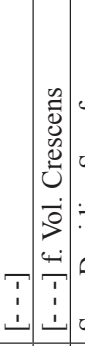 & 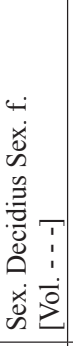 & 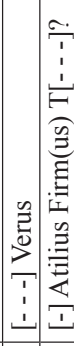 & 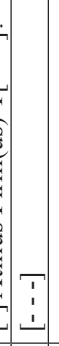 & 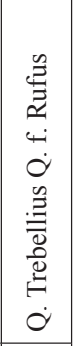 & \begin{tabular}{l}
$\bar{z}$ \\
$\bar{y}$ \\
\hdashline
\end{tabular} \\
\hline 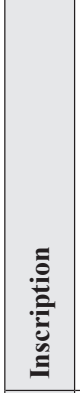 & 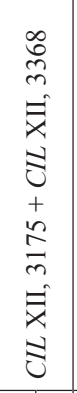 & 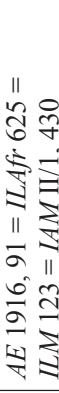 & 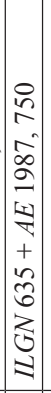 & $\begin{array}{l}\stackrel{े}{\vec{j}} \\
\tilde{m} \\
\vec{\nabla} \\
\vec{\Delta}\end{array}$ & 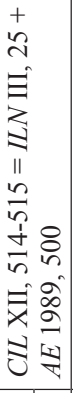 & 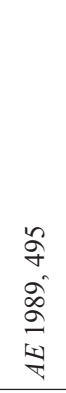 & 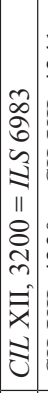 & 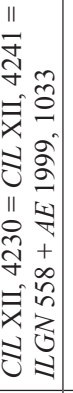 & 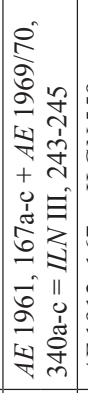 & 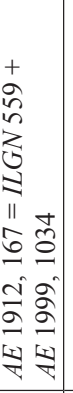 & & 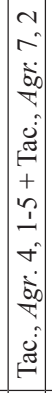 & 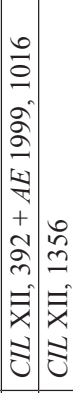 & 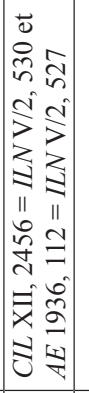 & 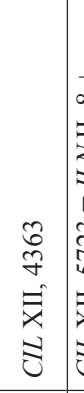 & 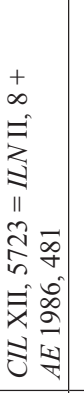 & 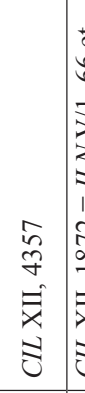 & 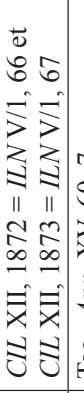 & 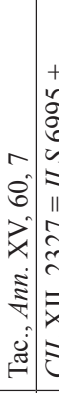 & 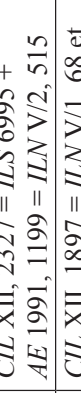 & 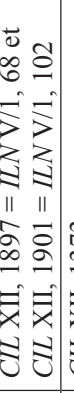 & 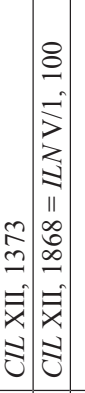 & 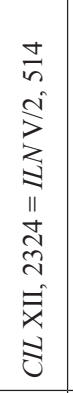 & 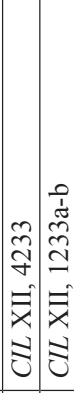 & 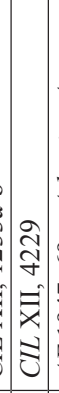 & 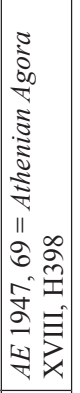 & ֻँ \\
\hline ż & 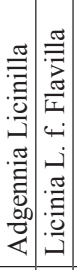 & 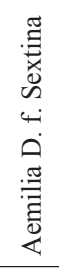 & 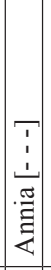 & 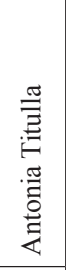 & 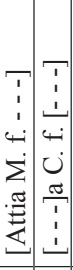 & $\begin{array}{l}\frac{\pi}{5} \\
\frac{\pi}{5} \\
\frac{\pi}{4} \\
\end{array}$ & 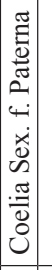 & 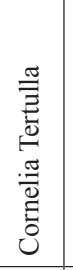 & 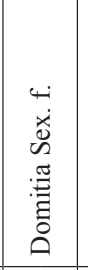 & 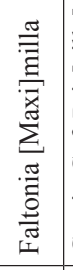 & 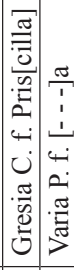 & & 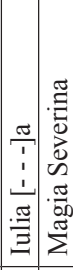 & 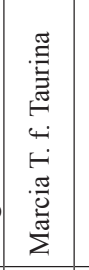 & 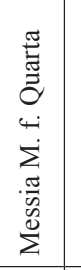 & 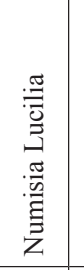 & 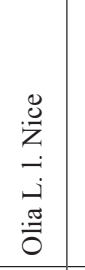 & 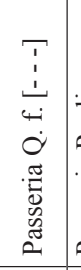 & 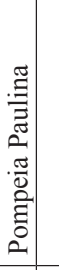 & 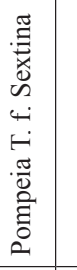 & 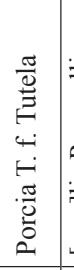 & 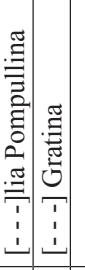 & 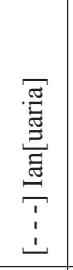 & 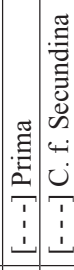 & $\begin{array}{c} \\
\vdots \\
\vdots \\
\vdots \\
\vdots\end{array}$ & 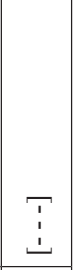 & \\
\hline & $-N$ & $m$ & + & in & $0 \wedge$ & $\infty$ & $a$ & $\stackrel{\circ}{\circ}$ & $=$ & $\simeq$ & $\cong \pm$ & & $\because=$ & $\stackrel{\infty}{-}$ & $\stackrel{2}{ }$ & กิ & $\vec{\sim}$ & ส & & d & त) & $\stackrel{\wedge}{\wedge}$ & $\stackrel{\infty}{\sim}$ & ते & $\vec{m}$ & $\approx \widetilde{m}$ & \\
\hline
\end{tabular}


décurion à Riez et décurion honorifique à Nîmes; L. Aponius [- - ], premier flamine d'Auguste puis préfet pro IIuiro de C. César à Béziers, après sa carrière équestre; [-] Domitius L. f. Vol. Macer, pontife à Aix-en-Provence; C. Gresius T. f. Pup. Domitus, édile, préfet pro Iluiro et flamine à Béziers; l'époux de Iulia [- - - ]a a détenu toutes les magistratures à Arles, puis après son passage sous les enseignes, il fut élu flamine provincial; le père de Messia M. f. Quarta, dont le surnom est Gallus fut édile et commissaire chargé du blé à Narbonne ainsi que flamine de Germanicus César, puis préfet des ouvriers et édile à Aix-en-Provence, tandis qu'un frère de Quarta, Senecio, à l'instar de Gallus, fut édile et commissaire chargé du blé; L. Aufidius L. f. Aem. Vinicianus Epagatinus, pour sa part, après la préfecture des ouvriers et une milice, fut édile et quinquennal à deux reprises non à Narbonne où il s'était installé, mais à Fundi, sa patrie; C. Passerius Q.f. Vol. Afer fut pour sa part IIIIvir, flamine du divin Auguste, puis de Germanicus à Vienne; également à Vienne, T. Pompeius T. f. Trom. Albinus avait été IIvir, avant de se lancer dans une carrière équestre; toujours dans la même cité, T. Porcius T. f. Vol. Latinus fut IIvir chargé du trésor et enfin IIIvir des domaines publics; à Vaison, l'époux anonyme de [- - -]lia Pompullina fut flamine du divin Auguste et pontife; Crescens, époux de Gratina, de Vienne, fut IIvir chargé du trésor et patron de la cité; Verus, mari ou père de Prima fut flamine de Rome et de l'Auguste à Béziers, tandis que, pour finir, Q. Trebellius Rufus, a accompli un cursus dans son entièreté à Toulouse, avant d'être élu flamine provincial et de partir pour Athènes, où il obtint des prêtrises et l'archontat éponyme.

Si leurs parents ont détenu des fonctions locales et des sacerdoces, les matrones équestres ne furent pas en reste (tabl. 3). En effet, plusieurs d'entre elles ont été élues comme flaminiques, ce qui atteste, par la même occasion, leur implication dans la vie municipale. C'est ainsi le cas de Aemilia D. f. Sextina, flaminique par deux fois, non pas à Vienne, d'où elle était originaire, mais à Volubilis, où elle s'était installée avec son mari; Cornelia Tertulla, flaminique de la colonie de Béziers; Licinia L. f. Flavilla, flaminica Augustae à Nîmes ; [- - - Gratina, flaminica Viennae; à Béziers encore, nous avons l'épouse anonyme d'un procurateur inconnu désignée à cette fonction, tout comme la femme de Q. Trebellius Rufus, prêtresse de Rome à Toulouse. A noter que hormis Cornelia Tertulla, toutes les autres femmes furent choisies sans que leurs proches n'aient été élevés, pour autant que nous le sachions, à la dignité sacerdotale.
Les renseignements que fournit le catalogue des matrones équestres de Narbonnaise ne s'arrêtent pas là : en effet, certaines inscriptions n'ont pas été mises au jour dans les centres urbains des cités où elles vécurent, ce que l'on peut expliquer de deux façons. Tout d'abord, citons Domitia Sex. f. et ses trois fils D. Domitius L. f. Vol. Celer, [-] Domitius L. f. Vol. Macer et L. Domitius L. f. Vol. Magu[- - -], dont seuls Celer et Macer furent officiers, qui sont connus par un monument funéraire découvert à Rognes : compte tenu de l'éloignement, une vingtaine de kilomètres, d'Aix-en-Provence, où Macer fut sans doute pontife, il faut considérer que les Domitii y possédaient une propriété où ils choisirent de se faire inhumer. Leur origine aixoise ne doit pas faire de doute, puisque c'est à la tribu Voltinia qu'étaient rattachés les trois fils Domitii et c'est sur le territoire de cette cité que fut mis au jour le mausolée (Burnand 1975). D'autres domaines sont documentés, dans des contextes analogues, comme à Cuers, sur le territoire d'Arles, où furent enterrés Iulia [- - - ]a et son mari, pratiquement anonyme; à Montmélian, Pompeia T. f. Sextina a érigé un cénotaphe en souvenir de son père ou de son frère dont le formulaire est pratiquement similaire à une inscription d'Augusta Emerita (Mérida), capitale de la Lusitanie, où T. Pompeius T. f. Trom. Albinus avait en fait fini ses jours ( $A E$ 1935, $5=$ ERAE 110). La tribu Tromentina présente dans l'onomastique d'Albinus semble indiquer une origine étrangère, d'Italie ou de Dalmatie, de cette famille. C'est toutefois à Vienne qu'Albinus fut IIvir. Le dernier témoignage dans cette catégorie nous est fourni par [- - - I Ianuaria, épouse de l'officier Sex. Decidius Sex. f. [- - ], tous deux connus par une inscription très endommagée de Notre-Dame-des-Millières, relevant de Vienne et où le couple était possessionné. Les sources littéraires ne sont pas en reste, puisque nous apprenons par Suétone qu'Iulia Procilla, mère de Cn. Iulius Agricola et native de Fréjus, avait été assassinée en 69 par les troupes d'Othon venues saccager sa propriété de Vintimille. Quant à Pompeia Paulina, nous savons qu'elle résidait dans une uilla suburbana des environs de Rome, sans qu'on puisse affirmer si elle appartenait à sa famille ou à son mari (tabl. 4).

\begin{tabular}{|c|c|c|}
\hline Nom & Fonction & Lieu \\
\hline Aemilia D. f. Sextina & Bis flaminica & Volubilis \\
\hline Cornelia Tertulla & Flaminica coloniae & Baeterrae \\
\hline Licinia L. f. Flavilla & Flaminica Augustae & Nemausus \\
\hline [- - - ] Gratina & Flaminica Viennae & Vienna \\
\hline$[---]$ & Flaminica & Baeterrae \\
\hline$[---]$ & 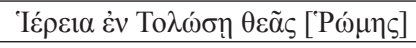 & Tolosa \\
\hline \multicolumn{3}{|c|}{ Tableau 3} \\
\hline
\end{tabular}




\begin{tabular}{|l|l|l|l|}
\hline Nom & Localisation du domaine & Cité de rattachement & Origo \\
\hline Domitia Sex. f. et ses fils & Rognes & Aquae Sextiae & Arelate \\
\hline Iulia [- - ]a et son mari & Cuers & Arelate & Arelate \\
\hline Pompeia T. f. Sextina et son père & Montmélian & Vienna & Vienna \\
\hline$[-$ - ] Ian[uaria] et son mari & Notre-Dame-des-Millières & Vienna & Forum Iulii \\
\hline Iulia Procilla & Albintimillium & Albintimillium & Arelate \\
\hline Pompeia Paulina & Ager Romanus & Roma \\
\hline \multicolumn{2}{|r}{ Tableau 4 } \\
\\
Liste des matrones équestres disposant de domaines fonciers.
\end{tabular}

\begin{tabular}{|l|l|l|}
\hline Nom & Lieu d'installation & Cité d'origine \\
\hline Aemilia D. f. Sextina et son mari & Volubilis & Vienna \\
\hline [Attia M. f. - - ] et sa famille & Aquae Sextiae & Arelate \\
\hline Coelia Sex. f. Paterna et son fils & Nemausus & Reii \\
\hline Iulia Procilla, son mari et son fils & Roma & Forum Iulii \\
\hline Iulia [- - ]a et son mari & Narbo Martius & Arelate \\
\hline Messia M. f. Quarta et sa famille & Narbo Martius & Aquae Sextiae \\
\hline Magia Severina et son frère & Vasio & Vercellae? \\
\hline Olia L. l. Nice et son mari & Narbo Martius & Fundi? \\
\hline Pompeia Paulina, son père et son frère & Roma & Arelate \\
\hline [- - ], son mari Q. Trebellius Rufus et son fils & Narbo Martius/Roma/Athenae & Tolosa \\
\hline \multicolumn{2}{|l}{ Tableau 5 } & \\
\hline
\end{tabular}

Puisque ces biens fonciers se trouvent à une distance parfois importante du centre urbain auquel ils sont administrativement rattachés, sauf exceptions, cela implique que ces dames se déplaçaient d'un endroit à un autre, quelquefois très éloigné (tabl. 5). C'est d'Arles que provenaient Attia et les siens, inscrits dans la tribu Teretina, mais installés à Aix-en-Provence; c'est sans doute à Riez qu'avait vu le jour Coelia Sex. f. Paterna, qui dressa l'autel funéraire de son fils à Nîmes, où il avait été fait décurion honoraire. On ne sait en revanche si elle l'y avait accompagné; Messia M. f. Quarta et sa famille, issue d'Aix-en-Provence, comme le confirme la tribu Voltinia de l'un d'entre eux, s'est finalement déplacée à Narbonne, d'où provient l'inscription qui les mentionne. Venue peutêtre d'au-delà des Alpes, de Verceil, pour être précis, Magia Severina est inhumée à Vaison, où son époux, Aurelius Valerianus lui érige une épitaphe au formulaire déficient, du point de vue de sa syntaxe et de son orthographe. Un doute aussi surgit dans le cas d'Olia L. 1. Nice, connue à Narbonne, mais dont le conjoint était originaire de Fundi. Elle pouvait s'y être unie avec lui et l'avoir ensuite suivi dans le sud de la Gaule. D'autres témoignages peuvent être invoqués, tels que celui de l'épouse anonyme de Q. Trebellius Rufus, dont j'ai dit qu'elle l'accompagna à Athènes, ou bien encore Aemilia D. f. Sextina, Viennoise décédée à Volubilis, où les derniers honneurs lui sont rendus: son cas est emblématique, dans la mesure où c'est elle qui confirme que son mari, Nammius Maternus, était également gaulois, et parce qu'elle avait détenu par deux fois un sacerdoce dans ce municipe de Maurétanie Tingitane, province où se situait le lieu de cantonnement de l'unité commandée par Maternus et où elle mourut. C'est en raison de son eximia probitas et des merita de son époux qu'elle se voit ainsi honorée par l'ordo de Volubilis (Hamdoune 2001). Autre exemple, celui de Pompeia Paulina, originaire d'Arles, mais installée à Rome où elle allait épouser Sénèque, illustre sénateur de Bétique. Enfin, quelques rares matrones ont, par la force des choses, dû effectuer un déplacement à un moment donné de leur vie: Iulia Procilla, vraisemblablement venue à Rome à la suite de son mari, L. Iulius L. f. Ani. Graecinus, sénateur prétorien, ainsi que Iulia [- - - ]a et l'épouse de Q. Trebellius Rufus, toutes deux mariées à des prêtres provinciaux, qu'elles accompagnèrent sans doute à Narbonne.

Que sait-on enfin de l'extraction de toutes ces femmes? A y regarder de plus près, toutes furent citoyennes romaines et porteuses d'anthroponymes tantôt pleinement latins, si l'on peut dire, comme Cornelia Tertulla, p. ex., ou bien d'origine «indigène». C'est le cas, plus précisément, d'Adgennia Licinilla de Nîmes, colonie latine, rappelons-le, ou d'Audasia, de Narbonne, sans oublier Passeria Q. f. [- - ] de Vienne ou les Messii d'Aix, bien qu'établis 
à Narbonne. Il demeure toutefois un exemple particulier, qui reflète une pratique minoritaire mais non moins réelle: la présence d'affranchies parmi les épouses de chevaliers. Je pense en particulier à Olia L. 1. Nice, qui affiche clairement sa condition d'ancienne esclave. Bien que son mari soit natif de Fundi, où il avait exercé des fonctions locales, il est possible, pour sa part, qu'elle soit originaire de la capitale provinciale, où d'autres Olii sont connus. Quoi qu'il en soit, une union entre un chevalier et une affranchie, loin d'être une norme, n'en reste pas moins une réalité, pour laquelle, il faut bien le reconnaître, il n'est guère loisible de percevoir les raisons. Il en va de même pour ces matrones, nées dans une famille «équestre», qui épousèrent des sénateurs: je songe ici à Iulia Procilla et à Pompeia Paulina, dont le propre frère fut d'ailleurs sénateur homo nouns. Filles toutes deux de hauts fonctionnaires, elles s'unirent à des homines noui, à ceci près que, contrairement à l'usage, Iulia Procilla s'est mariée à un homme de sa région. En effet, les alliances entre filles de chevaliers et sénateurs, généralement de première génération, sont doublement exogames: socialement et géographiquement, comme je l'ai montré dans ma thèse sous presse (Álvarez Melero s.p.).

Ainsi, le panorama que nous offrent les matrones équestres de Narbonnaise est très ample, comparé à d'autres provinces. On a pu se rendre compte qu'elles détenaient des sacerdoces, qu'elles possédaient des biens fonciers qu'elles devaient gérer en l'absence de leurs proches et qu'elles voyageaient de temps à autres. Toutefois, si les renseignements collectés jusqu'à présent nous autorisent donc à retracer certains aspects de leur vie quotidienne, pour ainsi dire, il subsiste des incertitudes: dans quelle mesure ontelles contribué à la promotion de leurs proches dans les ordres supérieurs? Si l'épigraphie laisse entrevoir qu'elles disposaient d'une richesse tirée de l'exploitation de leurs domaines qui leur permettaient de faire face aux coûts engendrés par l'élection au flaminicat, pour ne rien dire des héritages, p. ex., les sources littéraires, pour leur part, ne sont guère plus disertes à ce propos. Je pense en particulier à la question du patronage ou à la place des femmes dans les grands circuits économiques. Nous savons certes que Iulia Procilla appartenait à la fine fleur de l'aristocratie de Fréjus, fille d'un procurateur, représentant l'equestris nobilitas, pour paraphraser Tacite (Tac., Agr. 4, 1), mariée à un sénateur prétorien au destin tragique et qu'elle veilla étroitement à l'éducation de son fils, avant de périr assassinée suite aux vicissitudes de la guerre civile de 69. Bien que les renseignements à son endroit nous soient fournis par une source de première main, Tacite, nous ignorons si elle a joué un quelconque rôle dans la carrière de son mari puis de son fils. De même, les données dont nous disposons ne nous permettent malheureusement pas d'identifier les descendants de ces familles audelà d'une génération, sauf notables exceptions, dont celle de Iulia Procilla. A l'inverse, il n'est également pas possible d'élucider la place des antécédents familiaux des familles sénatoriales de Narbonnaise, si l'on en vient à examiner les données relatives au premier ordre: par exemple, le père de Pompeia Plotina, unie à un sénateur de Bétique à l'instar de Pompeia Paulina, était-il aussi de rang équestre? De fait, le cas de Pompeia Paulina est sans doute l'un des plus instructifs, dans la mesure où il atteste un mariage entre une fille d'un préfet de l'Annone (mais sœur de sénateur homo nouus), mariée à Sénèque, également homo nouus. En outre, cette union, qui constitue une belle promotion pour la famille de Paulina, est doublement exogamique compte tenu du rang équestre de son père, comparé à celui de Sénèque, mais aussi à cause de l'origine provinciale des époux, entre la Narbonnaise et la Bétique.

En conclusion, cette courte présentation des matrones équestres de Narbonnaise aura permis de cerner précisément un groupe de femmes provenant d'un milieu privilégié, nous éclairant sur leur conduite, leur visibilité spatiale remarquable, les pratiques matrimoniales et les sources de leur richesse. Il reste toutefois des questions que seule une étude globale permet partiellement de tirer au clair, puisque les femmes sont doublement discriminées, dans nos sources et par la loi: elles restent condamnées à jouer un rôle secondaire, dans l'ombre de leurs parents membres de l'uterque ordo. Malgré tout, et j'en reviens à R. Syme, l'étude des femmes n'en reste pas moins un seductive topic (Syme 1986, 168).

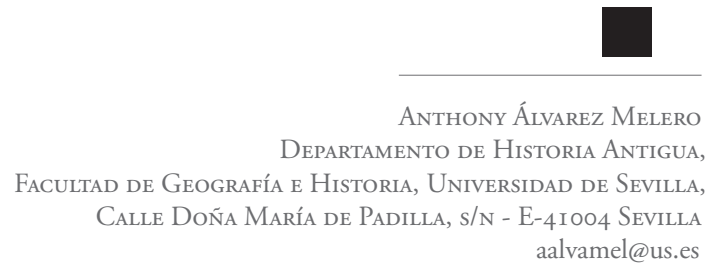




\section{Note de commentaire}

1. Ce travail a été mené dans le cadre des Projets de I+D, «Funciones y vínculos de las elites municipales de la Bética. marco jurídico, estudio documental y recuperación contextual del patrimonio epigráfico. I» (ORDO V) (Référence: HAR2014-55857-P) et «Marginación politica, jurídica y religiosa de la mujer durante el alto Imperio romano (siglos I-III)» (Référence: HAR201452725-P) du "Programa Estatal de Fomento de la Investigación Científica y Técnica de Excelencia del Ministerio de Economía y Competitividad", cofinancé par le Fonds Européen de Développement Régional (FEDER). 


\section{Références bibliographiques}

\section{Abréviations usuelles}

FOS : RAEPSAET-CHARLIER (M.-Th.) - Prosopographie des femmes de l'ordre sénatorial ( ${ }^{e r}$ $I I^{e}$ siècle). Louvain, Peeters, 1987 (Académie Royale de Belgique. Fonds René Draguet, 4).

FS: RÜPKE (J.) - Fasti sacerdotum. Die Mitglieder der Priesterschaften und das sakrale Funktionspersonal römischer, griechischer, orientalischer und jüdisch-christlicher Kulte in der Stadt Rom von 300 v. Chr. bis 499 n. Chr. Stuttgart, Franz Steiner, 2005 (Potsdamer altertumswissenschaftlicher Beiträge, 12).

$\boldsymbol{P I R}^{2}$ : GROAG (E.), STEIN (A.) et al. - Prosopographia Imperii Romani. Berlin, De Gruyter, 1933-2015.

\section{Bibliographie}

Álvarez Melero 2013 : ÁLVAREZ MELERO (A.) Los praefecti fabrum oriundos de las provincias hispanas. Una nueva aproximación. ETF(Ant.), $26,2013,123-154$

Álvarez Melero s.p. : ÁLVAREZ MELERO (A.) Matronae equestres. La parenté féminine des chevaliers romains originaires des provinces occidentales sous le Haut-Empire romain (I ${ }^{\text {er }}$ III ${ }^{e}$ siècles). Alliances matrimoniales, pratiques religieuses et mobilité géographique de femmes de l'élite romaine. Bruxelles-Rome, Brepols, sous presse.

Burnand 1975 : BURNAND (Y.) - Domitii Aquenses. Une famille de chevaliers romains de la région d'Aix-en-Provence: mausolée et domaine. Paris, De Boccard, 1975 (Suppl. à la RAN, 5).

Burnand 2005-2010: BURNAND (Y.) - Primores Galliarum. Sénateurs et chevaliers romains originaires de Gaule de la fin de la République au III ${ }^{e}$ siècle, I-IV. Bruxelles, Latomus, 2005-2010 (Coll. Latomus 290, 302, 306, 319, 328).
Christol 1993 : CHRISTOL (M.) - Les colonies de Narbonnaise et l'histoire sociale de la province. In: ECK (W.) éd. - Prosopographie und Sozialgeschichte. Studien zur Methodik und Erkenntnismöglichkeit der kaiserzeitlichen Prosopographie. Kolloquium Köln 24. 26. November 1991. Cologne-Vienne-Weimar, Böhlau, 1993, 277-291.

Christol 1999: CHRISTOL (M.) - La municipalisation de la Gaule Narbonnaise. In: DONDIN PAYRE (M.), RAEPSAET-CHARLIER (M.-Th.) éds. - Cités, colonies et municipes. Les processus de municipalisation en Gaule et en Germanie sous le Haut Empire romain. Paris, Publications de la Sorbonne, 1999, 1-27 (Histoire ancienne et médiévale, 53).

Christol 2009: CHRISTOL (M.) - L'ordre équestre en Narbonnaise: un groupe et ses personnalités entre cités, province et État. In : CHAUSSON (F.) éd. - Occidents romains. Sénateurs, chevaliers, militaires, notables dans les provinces d'Occident (Espagnes, Gaules, Germanies, Bretagne). Arles, Éditions Errance, 2009, 81-109.

Christol 2010 : CHRISTOL (M.) - Une histoire provinciale. La Gaule Narbonnaise de la fin du II siècle av. J.-C. au III siècle ap. J.-C. Scripta varia. Travaux édités avec le concours de BONSANGUE (M. L.) et HÖET-VAN CAUWENBERGHE (C.). Paris, Publications de la Sorbonne, 2010 (Histoire ancienne et médiévale, 103).

Demougin 1988 : DEMOUGIN (S.) - L'ordre équestre sous les Julio-Claudiens. Rome, École française de Rome, 1988 (Coll. de l'École française de Rome, 108).

Hamdoune 2001: HAMDOUNE (C.) - Une Gauloise à Volubilis. In: HAMDOUNE (C.) éd. Ubique amici. Mélanges offerts à Jean-Marie Lassère. Montpellier, Université Paul-Valéry, CERCAM, 2001, 225-237.
Pflaum 1978: PFLAUM (H.-G.) - Les fastes de la province de Narbonnaise. Paris, CNRS, 1978 (Gallia, Suppl. 30).

Pighi 1965: PIGHI (G.B.) - De ludis saecularibus populi Romani Quiritium libri sex. Amsterdam, P. Schippers, $1965^{2}$ (Pubblicazioni dell'Università cattolica del S. Cuore. Ser. 5. Scienze filologiche, vol. XXXV).

Raepsaet-Charlier 1987 : RAEPSAET-CHARLIER (M.-Th.) - Prosopographie des femmes de l'ordre sénatorial (I ${ }^{e r}-I I^{e}$ siècle). Louvain, Peeters, 1987 (Académie Royale de Belgique. Fonds René Draguet, 4).

Raepsaet-Charlier 1998 : RAEPSAET-CHARLIER (M.-Th.) - Les Gaules et les Germanies. In LEPELLEY (C.) dir. - Rome et l'intégration de l'Empire (44 av. J.-C.-260 ap. J.-C.), Tome II, Approches régionales du Haut-Empire romain. Paris, PUF, 1998, 143-195.

Sablayrolles 1984: SABLAYROLLES (R.) - Les praefecti fabrum de Narbonnaise. RAN, 17, 1984, 239-247.

Schäfer 2000 : SCHÄFER (N.) - Die Einbeziehung der Provinzialen in den Reichsdienst in augusteischer Zeit. Stuttgart, Franz Steiner, 2000 (Heidelberger althistorische Beiträge und epigraphische Studien, 33).

Scheid, Granino Cecere 1999: SCHEID (J.) et GRANINO CECERE (M.G.) - Les sacerdoces publics équestres. In: DEMOUGIN (S.), DEVIJVER (H.), RAEPSAET-CHARLIER (M.-Th.) éds. - L'ordre équestre. Histoire d'une aristocratie (II siècle av. J.-C.-III siècle ap. $J .-C$.). Actes du colloque international organisé par Ségolène Demougin, Hubert Devijver et Marie-Thérèse Raepsaet-Charlier (BruxellesLeuven, 5-7 octobre 1995). Rome, École française de Rome, 1999, 79-189 (Coll. de l'École française de Rome, 257).

Syme 1986: SYME (R.) - The Augustan Aristocracy. Oxford, Clarendon Press, 1986. 